\title{
Caudal fluoroscopy to guide venous access for pacemaker device implantation: should this now be standard practice?
}

\author{
Hitesh C Patel, ${ }^{1,2}$ Carl Hayward, ${ }^{3}$ Shane Nanayakkara, ${ }^{1,2}$ Archer Broughton, ${ }^{2}$ \\ Justin A Mariani, ${ }^{1,2}$
}

${ }^{1}$ Heart Failure Research Group, Baker IDI Heart and Diabetes Research Institute, Melbourne, Victoria, Australia

${ }^{2}$ Heart Centre, Alfred Hospital, Melbourne, Victoria, Australia ${ }^{3}$ Barts Heart Centre, St Bartholomew's Hospital, London, UK

\section{Correspondence to}

Dr Hitesh C Patel, Heart Centre, Alfred Hospital, 55 Commercial Road, Melbourne, VIC, 3004, Australia; dochiteshpatel@hotmail.com

Received 15 January 2017 Revised 15 February 2017 Accepted 16 February 2017

\section{ABSTRACT}

We describe a technique that uses both posterioranterior and caudal fluoroscopy to achieve venous access for pacemaker device implantation. A significant advantage of this technique is the ability to clearly demarcate both the anatomy of venous drainage and the lung border. We would encourage all centres to adopt this technique as a safe approach to venous access.

\section{INTRODUCTION}

The subclavian (extrathoracic or intrathoracic) vein is used for venous access in at least $40 \%$ of cardiac rhythm management device implants. ${ }^{1}$ It courses from the lateral border of the first rib to the sternal end of the clavicle (figure 1A, B). The advantage of using this vein is that it is safely accessible from a percutaneous approach and large enough to accommodate multiple leads. The pneumothorax risk reported in clinical trials when using this vein is reported as $1.1 \% .^{2}$ The section of the subclavian vein that lies over the first rib is preferred by some operators as this region is theoretically less prone to subclavian crush and, being extrathoracic, has a lower risk of pneumothorax.

The subclavian vein can be accessed by a variety of techniques but the two most commonly employed are: blind percutaneous puncture using palpable anatomical landmarks or fluoroscopically guided puncture in the posterior-anterior (PA) projection.

In this Clinical Practice report, we describe our experience of combining PA and caudal fluoroscopy to achieve subclavian vein access.

\section{TECHNIQUE OF COMBINING PA AND CAUDAL FLUOROSCOPY TO ACHIEVE VENOUS ACCESS}

This technique can be used with any conventional incision (deltopectoral groove, horizontal or oblique). A standard puncture needle attached to a $5 \mathrm{~mL}$ syringe is placed on top of the prepectoral fascia and positioned along the likely path of the axillary vein, approximately $60^{\circ}-70^{\circ}$ from the craniocaudal axis with the target for puncture being the area between the inferior aspect of the clavicle and the first rib (figure 1B). This puncture can be facilitated with contrast venography although not necessary. In this orientation, the bevel of the needle is then inserted $5 \mathrm{~mm}$ into the prepectoral fascia between the first and second rib, at an angle of $35^{\circ}-60^{\circ}$ horizontally to the path of the vein, aiming for the intersection of the clavicle and first rib. The fluoroscopy camera is then moved to a caudal $35^{\circ}-45^{\circ}$ angle in order to assist in determining the depth of puncture. The intention is to aspirate venous blood by aiming for the landing zone anterior to the thoracic cage outline (white box in figure 1D) and avoid entering the thoracic cavity. If venous access is not obtained on first pass, the needle should be retracted until it is superficial and its trajectory adjusted initially to a steeper or shallower course. If access is still not achieved, the operator should return to the PA view and aim the needle more cranial or caudal but still towards the first rib/clavicle intersection; there should be a low threshold in performing a contrast venogram. If an inadvertent arterial puncture is performed then the needle should be withdrawn and a more inferiorly directed attempt made following haemostasis.

In patients with existing leads undergoing lead revisions or device upgrade, in whom the risk of complications are higher, ${ }^{3}$ the caudal view may also facilitate a safer puncture of the subclavian vein.

\section{OUR EXPERIENCE}

The described technique has been routine practice for two operators at our centre who are responsible for $76 \%$ of device implants. In the period from December 2011 to December 2016, there were 1589 cases requiring central venous access: 1051 pacemaker implants, 507 complex devices implants (defibrillators and/or biventricular pacemakers) and 25 lead revisions/device upgrades. The caudal view was used in 1207 (76\%) cases, the cephalic vein cut down in $274(17 \%)$ cases and standard PA fluoroscopy in $108(7 \%)$ cases. There were three pneumothoraces requiring drainage; in two patients these occurred despite using the caudal view and in one this occurred using PA fluoroscopy alone $(0.17 \%$ vs $0.93 \%$, Fisher's exact test, $p=0.23)$. The overall incidence of pneumothorax was $0.2 \%$, which is fivefold lower than that described in published trials and twofold lower than in registry data. ${ }^{2}$

\section{DISCUSSION}

We have described a technique that can improve ease and safety for venous access during cardiac rhythm device implantation that does not require additional equipment. The rate of pneumothorax in our institute is low compared with published trials and registries and we believe that our use of the described technique has a significant contributory role. ${ }^{4}$ Although we are not the first to report the use of this technique, our series is the largest published to date. ${ }^{5}$

The traditional technique of subclavian puncture using PA fluoroscopy does not allow perception of the depth of the needle which may be particularly 
Figure 1 Fluoroscopic images in the posterior-anterior (PA) and caudal projection with and without contrast. (A) Displays a venogram in the PA projection. Anatomical landmarks identified in the PA projection are identified in (B). (B) Also illustrates the target for venous puncture being the white box at the junction of the inferior border of the clavicle and the first rib. The craniocaudal alignment for access of $60^{\circ}-70^{\circ}$ from the vertical which lies above the expected path of the vein is also illustrated in (B). (C) Displays a caudal venogram. (D) Illustrates a caudal projection and demonstrates the course of the subclavian vein (a wire has been inserted through it) and the white box demarcates the ideal landing zone for a needle to puncture (there is a needle in the vein visible).
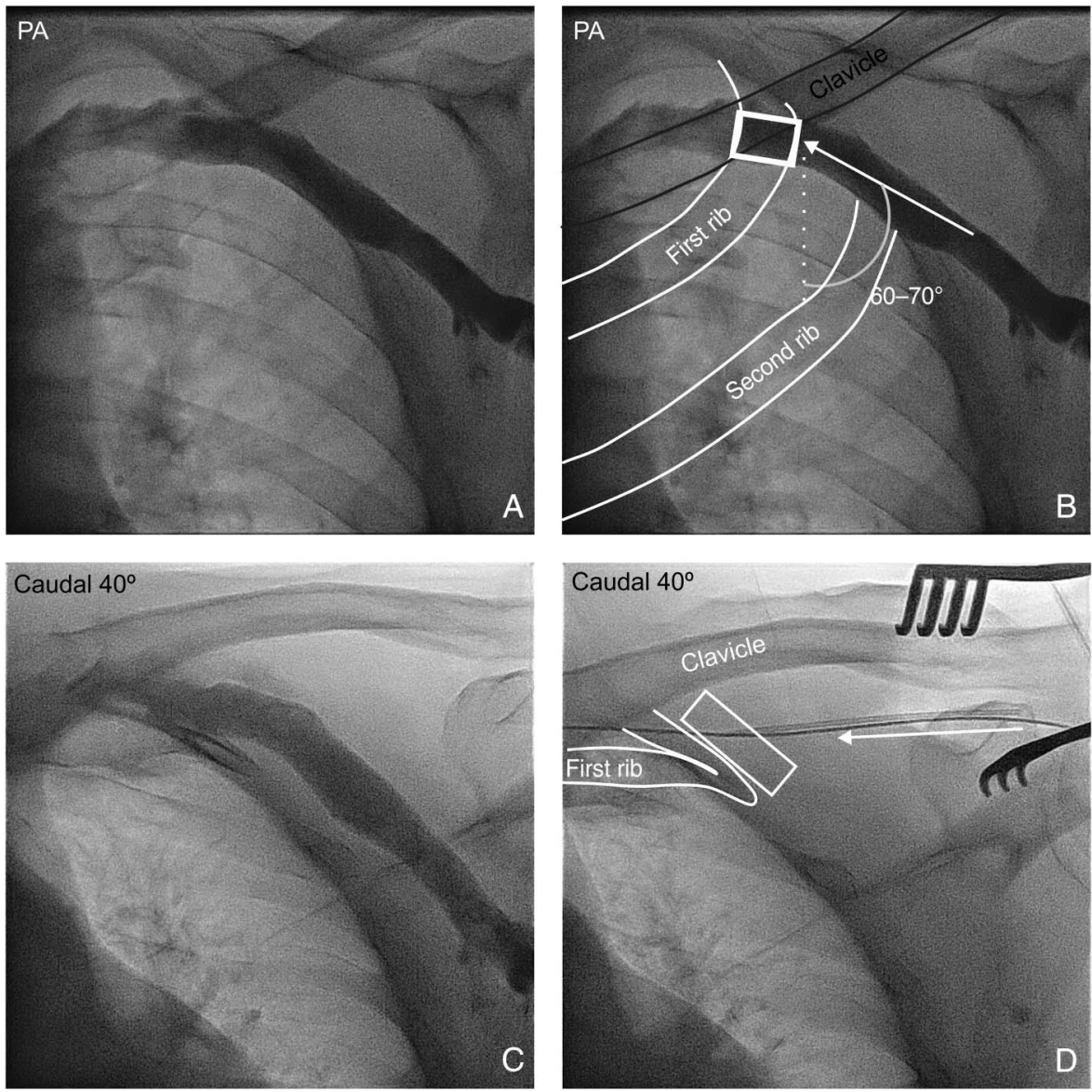

problematic in patients who either have a large body habitus or have altered thoracic cage anatomy (eg, patients with kyphoscoliosis or those with chronic obstructive lung disease with significant lung hyperinflation). In this situation, operators may choose to puncture the subclavian vein over the first rib using fluoroscopy in the PA projection. This technique is entirely dependent on being able to clearly visualise the patient's ribs on fluoroscopy, which can be challenging in some patients, particularly the elderly. Furthermore, in using the PA approach to puncture the subclavian vein over the first rib, many operators will chose a steep angle (perpendicular to the chest wall) to minimise any drift in the superior/inferior directions, which could lead to a puncture beyond the boundaries of the first rib and hence a pneumothorax. However, accessing the subclavian vein at a perpendicular angle can theoretically increase flexion stress on the lead. ${ }^{6}$ The caudal view improves visualisation of the first rib and lung borders (figure 1C) and permits a shallower angle of entry into the subclavian vein theoretically reducing the risk of flexion damage to the lead. Finally, performing the venous puncture in the caudal view removes the operator's hand from the field of view, reducing direct radiation exposure.

\section{CONCLUSION}

Combining PA and caudal fluoroscopic views while obtaining venous access for device implants may reduce the risk of pneumothorax.
Contributors HCP and JAM conceived this manuscript. HCP wrote the initial draft. All authors provided substantial input in writing the manuscript. All authors approved the final version.

Competing interests None declared.

Ethics approval Alfred Low Risk Ethics Committee.

Provenance and peer review Not commissioned; externally peer reviewed.

Data sharing statement Please contact the corresponding author by email if further information is required.

\section{REFERENCES}

1 Bongiorni MG, Proclemer A, Dobreanu D, et al. Preferred tools and techniques for implantation of cardiac electronic devices in Europe: results of the European Heart Rhythm Association survey. Europace 2013;15: 1664-8.

2 Ezzat VA, Lee V, Ahsan S, et al. A systematic review of ICD complications in randomised controlled trials versus registries: is our 'real-world' data an underestimation? Open Heart 2015;2:e000198.

3 Hildick-Smith DJ, Lowe MD, Newell SA, et al. Ventricular pacemaker upgrade: experience, complications and recommendations. Heart 1998;79:383-7.

4 Kirkfeldt RE, Johansen JB, Nohr EA, et al. Complications after cardiac implantable electronic device implantations: an analysis of a complete, nationwide cohort in Denmark. Eur Heart J 2014;35:1186-94.

5 Yang $F$, Kulbak G. A new trick to a routine procedure: taking the fear out of the axillary vein stick using the $35^{\circ}$ caudal view. Europace $2015 ; 17$ 1157-60.

6 Krahn AD, Morissette J, Lahm R, et al. Radiographic predictors of lead conducto fracture. Circ Arrhythm Electrophysiol 2014;7:1070-7. 